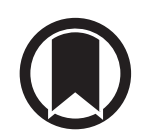

CrossMark

\title{
Transcriptome profiling reveals the complexity of pirfenidone effects in idiopathic pulmonary fibrosis
}

\author{
Grazyna Kwapiszewska ${ }^{1,2,12}$, Anna Gungl ${ }^{2}$, Jochen Wilhelm ${ }^{3,11}$, \\ Leigh M. Marsh (10) ${ }^{1}$, Helene Thekkekara Puthenparampil ${ }^{1}$, Katharina Sinn ${ }^{4}$, \\ Miroslava Didiasova ${ }^{5}$, Walter Klepetko ${ }^{4}$, Djuro Kosanovic ${ }^{3}$, Ralph \\ T. Schermuly ${ }^{3,11}$, Lukasz Wujak ${ }^{5}$, Benjamin Weiss ${ }^{6}$, Liliana Schaefer ${ }^{7}$, \\ Marc Schneider ${ }^{8,11}$, Michael Kreuter ${ }^{9,11}$, Andrea Olschewski ${ }^{1}$, \\ Werner Seeger ${ }^{3,11}$, Horst Olschewski ${ }^{1,10}$ and Malgorzata Wygrecka ${ }^{5,11,12}$
}

\begin{abstract}
Affiliations: ${ }^{1}$ Ludwig Boltzmann Institute for Lung Vascular Research, Graz, Austria. ${ }^{2}$ Otto Loewi Research Center, Medical University of Graz, Graz, Austria. ${ }^{3}$ Dept of Internal Medicine, Universities of Giessen and Marburg Lung Center, Giessen, Germany. ${ }^{4}$ Dept of Thoracic Surgery, Medical University of Vienna, Vienna, Austria. ${ }^{5}$ Dept of Biochemistry, Universities of Giessen and Marburg Lung Center, Giessen, Germany. ${ }^{6}$ Dept of Surgery, Universities of Giessen and Marburg Lung Center, Giessen, Germany. ${ }^{7}$ Goethe University School of Medicine, Frankfurt am Main, Germany. ${ }^{8}$ Translational Research Unit, Thoraxklinik University Hospital Heidelberg, Heidelberg, Germany. ${ }^{9}$ Center for Interstitial and Rare Lung Diseases Pneumology and Respiratory Critical Care Medicine, Thoraxklinik University of Heidelberg, Translational Lung Research Center Heidelberg (TLRC), Heidelberg, Germany. ${ }^{10}$ Dept of Pulmonology, Medical University of Graz, Graz, Austria.

${ }^{11}$ Members of the German Center for Lung Research. ${ }^{12}$ Joint lead authors.
\end{abstract}

Correspondence: Malgorzata Wygrecka, Dept of Biochemistry, Faculty of Medicine, Universities of Giessen and Marburg Lung Center, Friedrichstrasse 24, 35392 Giessen, Germany.

E-mail: malgorzata.wygreckađinnere.med.uni-giessen.de

@ERSpublications

Pirfenidone's mode of action in human lungs involves a complex interactome comprising genes related to inflammation and extracellular matrix architecture http://ow.ly/26NN30lpGON

Cite this article as: Kwapiszewska G, Gungl A, Wilhelm J, et al. Transcriptome profiling reveals the complexity of pirfenidone effects in idiopathic pulmonary fibrosis. Eur Respir J 2018; 52: 1800564 [https:// doi.org/10.1183/13993003.00564-2018].

ABSTRACT Despite the beneficial effects of pirfenidone in treating idiopathic pulmonary fibrosis (IPF), it remains unclear if lung fibroblasts (FB) are the main therapeutic target.

To resolve this question, we employed a comparative transcriptomic approach and analysed lung homogenates $(\mathrm{LH})$ and FB derived from IPF patients treated with or without pirfenidone.

In $\mathrm{FB}$, pirfenidone therapy predominantly affected growth and cell division pathways, indicating a major cellular metabolic shift. In LH samples, pirfenidone treatment was mostly associated with inflammationrelated processes. In FB and $\mathrm{LH}$, regulated genes were over-represented in the Gene Ontology node "extracellular matrix". We identified lower expression of cell migration-inducing and hyaluronan-binding protein (CEMIP) in both LH and FB from pirfenidone-treated IPF patients. Plasma levels of CEMIP were elevated in IPF patients compared to healthy controls and decreased after 7 months of pirfenidone treatment. CEMIP expression in FB was downregulated in a glioma-associated oncogene homologue-dependent manner and CEMIP silencing in IPF FB reduced collagen production and attenuated cell proliferation and migration.

Cumulatively, our approach indicates that pirfenidone exerts beneficial effects via its action on multiple pathways in both FB and other pulmonary cells, through its ability to control extracellular matrix architecture and inflammatory reactions.

This article has supplementary material available from erj.ersjournals.com

Received: March 212018 | Accepted after revision: Aug 032018

Copyright OERS 2018. This article is open access and distributed under the terms of the Creative Commons Attribution Licence 4.0. 


\section{Introduction}

Idiopathic pulmonary fibrosis (IPF) is a devastating disease with a median survival of $<5$ years following diagnosis and a mortality rate that exceeds many types of cancer. An IPF diagnosis leads to a high burden of morbidity with poor quality of life. In spite of recently approved medical therapies, lung transplantation still remains the only definitive treatment option [1].

The basic pathologic mechanisms in IPF are characterised by injury and activation of alveolar epithelial cells, (myo)-fibroblast proliferation and dysregulated transcriptional and post-translational control of extracellular matrix (ECM) components, such as collagen and hyaluronan (HA), in the lung. As a result, there are changes reminiscent of excessive scar formation, with irreversible damage to the lung architecture that causes disturbances in ventilation, perfusion and gas exchange and eventually death from cardiorespiratory failure [2].

Despite inhibitory effects on lung fibroblast (FB) proliferation and collagen production, several treatment strategies have failed to show a positive effect in IPF patients; these include interferon- $\gamma$ [3], the tyrosine kinase inhibitor imatinib [4] and the selective endothelin receptor antagonists bosentan [5] and ambrisentan [6]. Accordingly, a strong or conditional negative recommendation has been attributed to these therapeutic concepts in the current international clinical practice guidelines for IPF [7]. Currently, there are only two approved drugs for the treatment of IPF, pirfenidone and nintedanib [7]. Pirfenidone (5-methyl-1-phenyl-2(1H)-pyridinone) is an orally available synthetic drug that was approved in 2011 in Europe and in 2014 in the USA for the treatment of mild to moderate IPF. To date, four randomised placebo-controlled phase III studies have demonstrated that pirfenidone significantly slows down disease progression by reducing the decline in forced vital capacity (FVC) [8-11]. Combined data from the CAPACITY 1 and 2 as well as ASCEND trials showed a significant benefit with respect to progression-free survival, 6-min walk test and dyspnoea score $[8,9]$. Furthermore, pooled analysis of data from four phase III trials demonstrated a reduction in all-cause mortality under pirfenidone treatment [12]. In real-world observational studies of pirfenidone treatment of IPF, the effects of pirfenidone on FVC decline were consistent with the findings from the clinical trials [13].

Although recent findings demonstrated that pirfenidone exerts pleiotropic effects on numerous cell types $[14,15]$, the mechanisms by which pirfenidone slows IPF progression are not well understood. Here, by applying a complex unbiased transcriptomic approach employing 1) lung tissue from pirfenidone-treated and pirfenidone-naïve IPF patients, 2) isolated FB from the same patients and 3) cultured FB from IPF patients treated in vitro with pirfenidone, we found that pirfenidone exerts most of its effects on the lung by regulating the expression of genes involved in inflammation and ECM architecture.

\section{Methods}

A description of the methodologies is provided in the supplementary material; here, only information about IPF patients is given.

\section{Human lungs}

Human lung samples were obtained from patients with IPF who underwent lung transplantation at the Dept of Surgery, Division of Thoracic Surgery, Medical University of Vienna, Vienna, Austria. The protocol and tissue usage were approved by the institutional ethics committee (976/2010) and patient consent was obtained before lung transplantation. Microscopic sections were prepared and stained with hematoxylin and eosin to assess the morphology of the fibrotic lungs.

\section{Results}

\section{Study population}

Transplanted lungs from 14 clinically well-characterised patients with IPF were used in this study. Demographic characteristics and clinical data of the IPF patient experimental and validation cohorts, including pirfenidone (Esbriet ${ }^{\oplus} /$ Pirfenex) medication, are reported in table 1 . These parameters did not show significant differences between the treated and non-treated groups. All lungs possessed classical characteristics of parenchymal remodelling with the typical pattern of usual interstitial pneumonia (supplementary figure S1).

\section{Effects of pirfenidone on the expression profiles in lung homogenates and isolated fibroblasts}

To determine the effects of pirfenidone treatment on the molecular signature in situ, we performed gene expression profiling on 1) lung homogenate (LH) samples and 2) isolated human lung FB from pirfenidone-treated (P) or pirfenidone-naïve IPF patients. LH samples are heterogeneous tissue samples that can be used to identify individual genes that have a strong treatment-associated average change in gene expression, pointing to processes that are either directly or indirectly changed by pirfenidone. In 
TABLE 1 Demographics and clinical data of donors and IPF patients in the transcriptome cohort and the validation cohort

\begin{tabular}{|c|c|c|c|c|c|}
\hline \multirow[t]{2}{*}{ Variable } & \multicolumn{2}{|c|}{$\begin{array}{l}\text { Cohort used for } \\
\text { transcriptome } \\
\text { profiling }\end{array}$} & \multicolumn{3}{|c|}{ Validation cohort } \\
\hline & IPF & $I P F+P$ & Donor & IPF & $I P F+P$ \\
\hline Subjects & 7 & 7 & 31 & 23 & 7 \\
\hline Age years & $56.8 \pm 9.0$ & $55.4 \pm 7.5$ & $51.4 \pm 14.7$ & $61.8 \pm 12.9$ & $58.0 \pm 5.4$ \\
\hline \multicolumn{6}{|l|}{ Sex } \\
\hline Male & 4 & 5 & 12 & 16 & 4 \\
\hline Female & 3 & 2 & 19 & 7 & 3 \\
\hline \multicolumn{6}{|l|}{ Smoking status } \\
\hline Never & 0 & 2 & & 7 & 2 \\
\hline Former & 7 & 5 & & 16 & 5 \\
\hline Current & 0 & 0 & & 0 & 0 \\
\hline FVC \% pred & $39.6 \pm 16.7$ & $49.5 \pm 11.2$ & & $49.1 \pm 13.0$ & $48.1 \pm 10.2$ \\
\hline Histological confirmation of a UIP pattern $\%$ & 100 & 100 & & 100 & 100 \\
\hline Daily dose of pirfenidone $\mathbf{g}$ & & $2.0 \pm 0.7$ & & & $1.9 \pm 0.6$ \\
\hline Duration of pirfenidone treatment months & & $8.4 \pm 4.8$ & & & $11.2 \pm 3.8$ \\
\hline
\end{tabular}

Data are presented as $\mathrm{n}$ or mean $\pm \mathrm{SD}$, unless otherwise specified. IPF: idiopathic pulmonary fibrosis; P: pirfenidone; FVC: forced vital capacity; UIP: usual interstitial pneumonia.

contrast, analysing isolated FB could identify cell-type-specific responses to pirfenidone treatment. The experimental design is presented in figure 1a.

A comparison of the entire gene expression profile by principal component analysis (PCA) revealed only minor global changes between $\mathrm{LH}$ samples from pirfenidone-treated (IPF+P(LH)) and pirfenidone-naïve $(\operatorname{IPF}(\mathrm{LH})$ ) patients (figure 1b, i). Global gene set tests using the Kyoto Encyclopedia of Gene and Genomes (KEGG) showed several significantly perturbed pathways (figure $1 \mathrm{~b}$, ii). These included inflammatory processes and changes in cell-cell contact (e.g. tight junction, endocytosis). The global distribution of all genes according to the respective log fold changes (LFCs) and p-values are depicted in the volcano plot in figure $1 \mathrm{~b}$, iii, and figure $1 \mathrm{~b}$, iv shows the expression levels of the top 20 regulated genes at the single-patient level.

The expression profiles of $\mathrm{FB}$ isolated from pirfenidone-treated (IPF $+\mathrm{P}(\mathrm{FB}))$ and pirfenidone-naive (IPF $(\mathrm{FB})$ ) patients were distinct and gave good separation in PCA (figure 1c, i). The most significantly altered pathways in FB indicated that cells possessed general metabolic alterations related to growth and cell division (e.g. DNA replication, cell cycle) and modified protein turnover (e.g. proteasome) (figure 1c, ii). The top 20 regulated genes according to the p-value ranking are highlighted in the volcano plot in figure $1 \mathrm{c}$, iii, and single-patient-level expression is shown in figure 1c, iv.

Comparing $\mathrm{LH}(\mathrm{IPF}(\mathrm{LH}), \mathrm{IPF}+\mathrm{P}(\mathrm{LH}))$ and $\mathrm{FB}(\mathrm{IPF}(\mathrm{FB}), \mathrm{IPF}+\mathrm{P}(\mathrm{FB}))$ samples by PCA gave a clear separation that primarily discriminated between LH and FB (figure 2a). Global gene set tests showed a larger number of significantly perturbed pirfenidone-induced pathways in FB than in LH (figure $2 \mathrm{~b}$ ). This was expected because the profiles obtained from LH represent a mixture of the responses of many cell types, so that the cell-type-specific perturbation of defined pathways is more difficult to ascertain. Analysis of the 100 genes with the largest differences showed clear separation of LH and FB expression profiles in a hierarchical cluster analysis (Euclidean distance and complete linkage; figure 2c). Gene clustering revealed several groups of genes with a source-specific regulation associated with pirfenidone treatment (regulated in LH but not in FB (blue cluster), regulated in FB but not in LH (yellow cluster), oppositely regulated in LH and in FB (grey cluster) and concurrently regulated in LH and FB (green cluster)) (figure $2 \mathrm{c}$ and supplementary table $\mathrm{S} 1$ ).

\section{Pirfenidone induces distinct gene regulation in $\mathrm{LH}$ and isolated $\mathrm{FB}$}

To understand the transcriptional repertoire being specifically regulated in response to pirfenidone in either compartment, we selected genes regulated only in one group, either LH or FB. For this purpose, the threshold was set to a LFC $>|1.41|$ to define upregulated genes and $<|0.5|$ to define not regulated genes (coloured dots in figure 3a and listed as "only in LH/only in FB" in supplementary table S1). This way we focused on genes either induced by at least a twofold change or repressed to at least $50 \%$. Over-representation analysis of these selectively regulated genes with Gene Ontology (GO) nodes revealed that in FB, the selected genes were contained within the "extracellular matrix", "cytosol" and "nucleus" 
a)
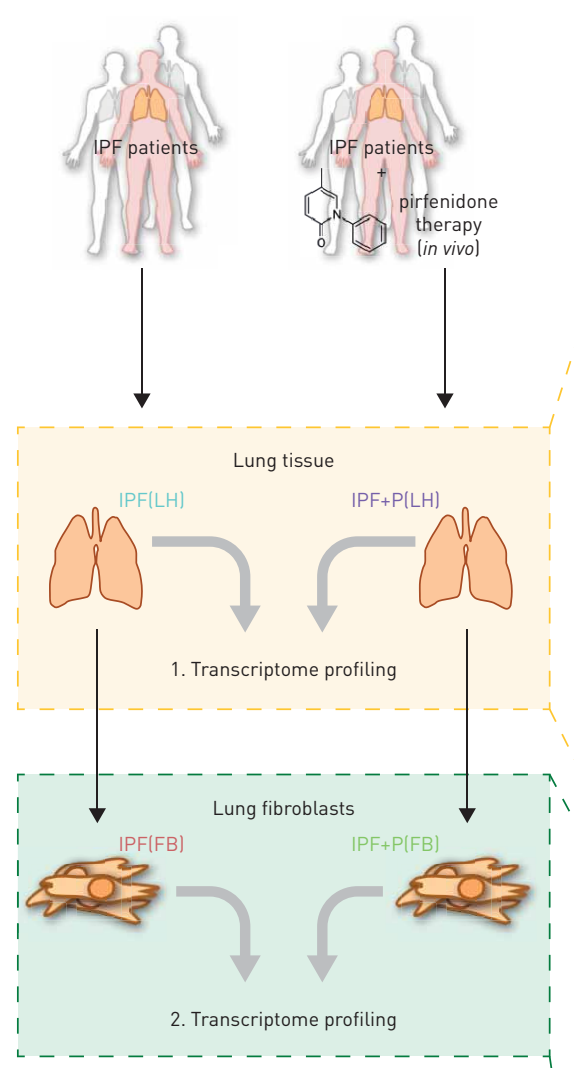

b)

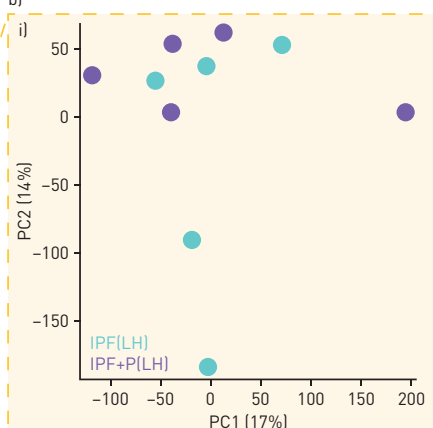

iii)

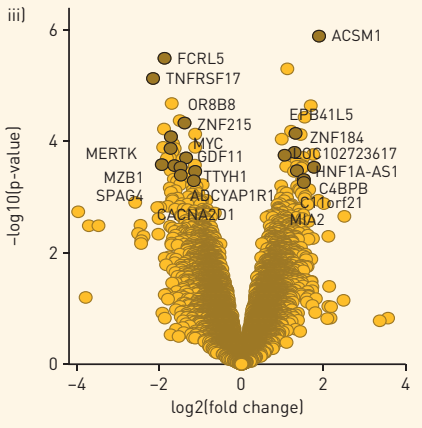

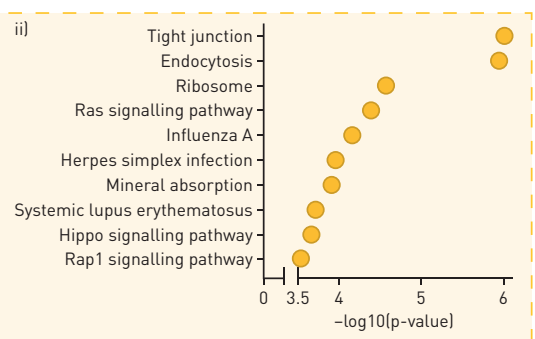

iv)

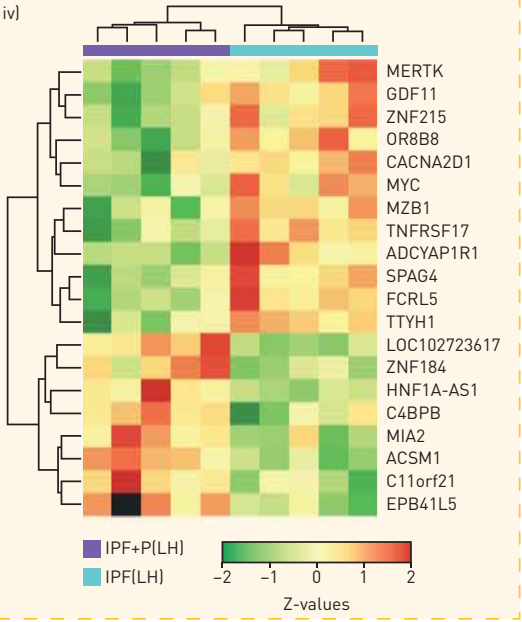

$$
\begin{gathered}
1 \\
\backslash \\
c \\
c
\end{gathered}
$$

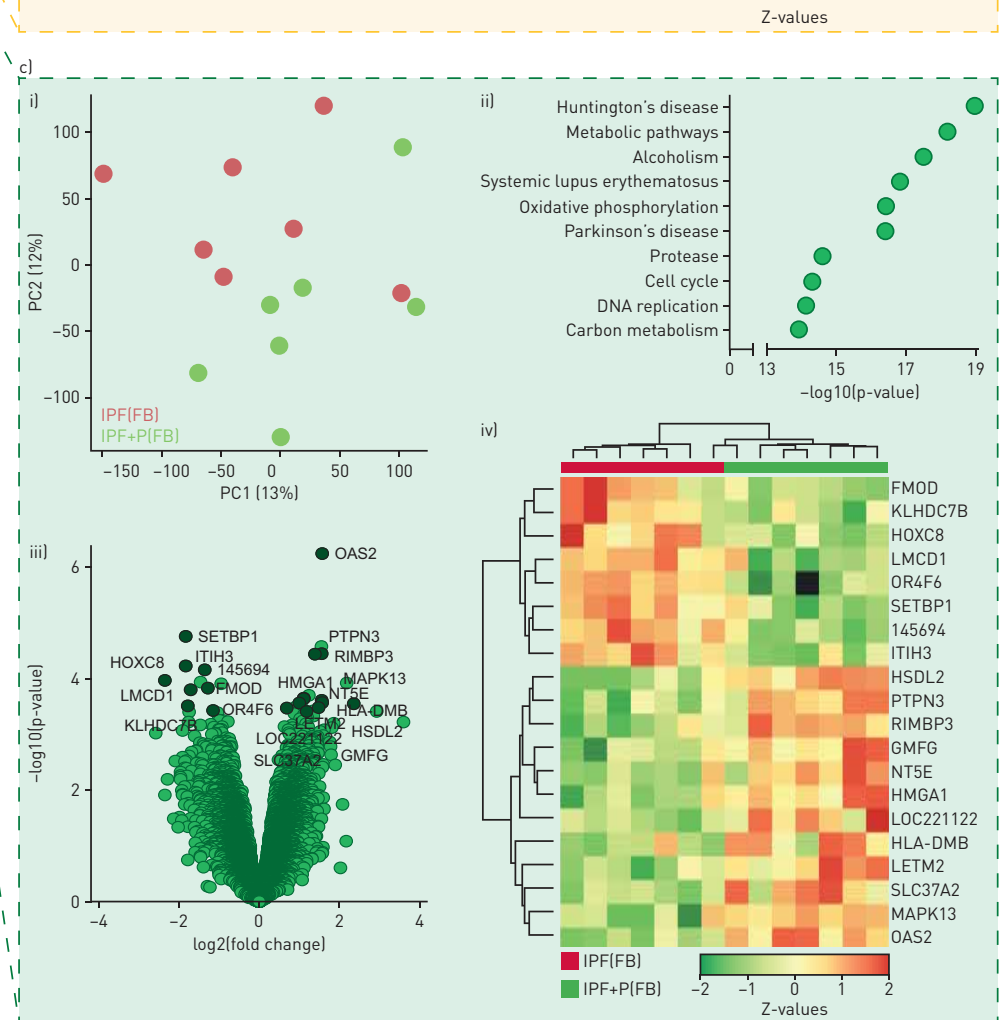

FIGURE 1 Transcriptomic profiling of human lung homogenates (LH) and human lung fibroblasts (FB) derived from idiopathic pulmonary fibrosis (IPF) patients treated with pirfenidone (P). a) Schematic overview of the experimental design. b) Transcriptome profiling of IPF LH. b, i) Principal

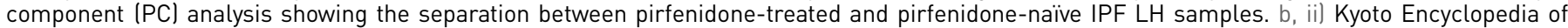
Gene and Genomes (KEGG)-based gene function analysis showing the top ten most affected pathways after pirfenidone treatment in IPF LH. b, iii) Volcano plot indicating the global distribution of log2 (fold change in expression) and p-value. The labelling shows the 20 genes with the highest significance. b, iv) Heat map showing the clustering between treated and non-treated samples for the top 20 regulated genes as in b, iii. c) Transcriptome profiling of IPF FB. C, i) PC analysis plot showing clear separation between pirfenidone-treated and pirfenidone-naïve IPF FB. C, ii) KEGG analysis showing the ten most significantly perturbed pathways in IPF FB after pirfenidone treatment. $c$, iii) Volcano plot indicating the

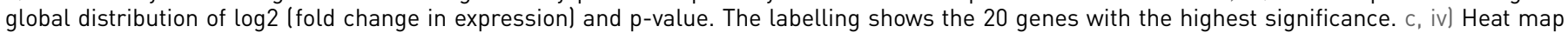
representing expression at single-patient level for the top 20 genes as in $c$, iii. 

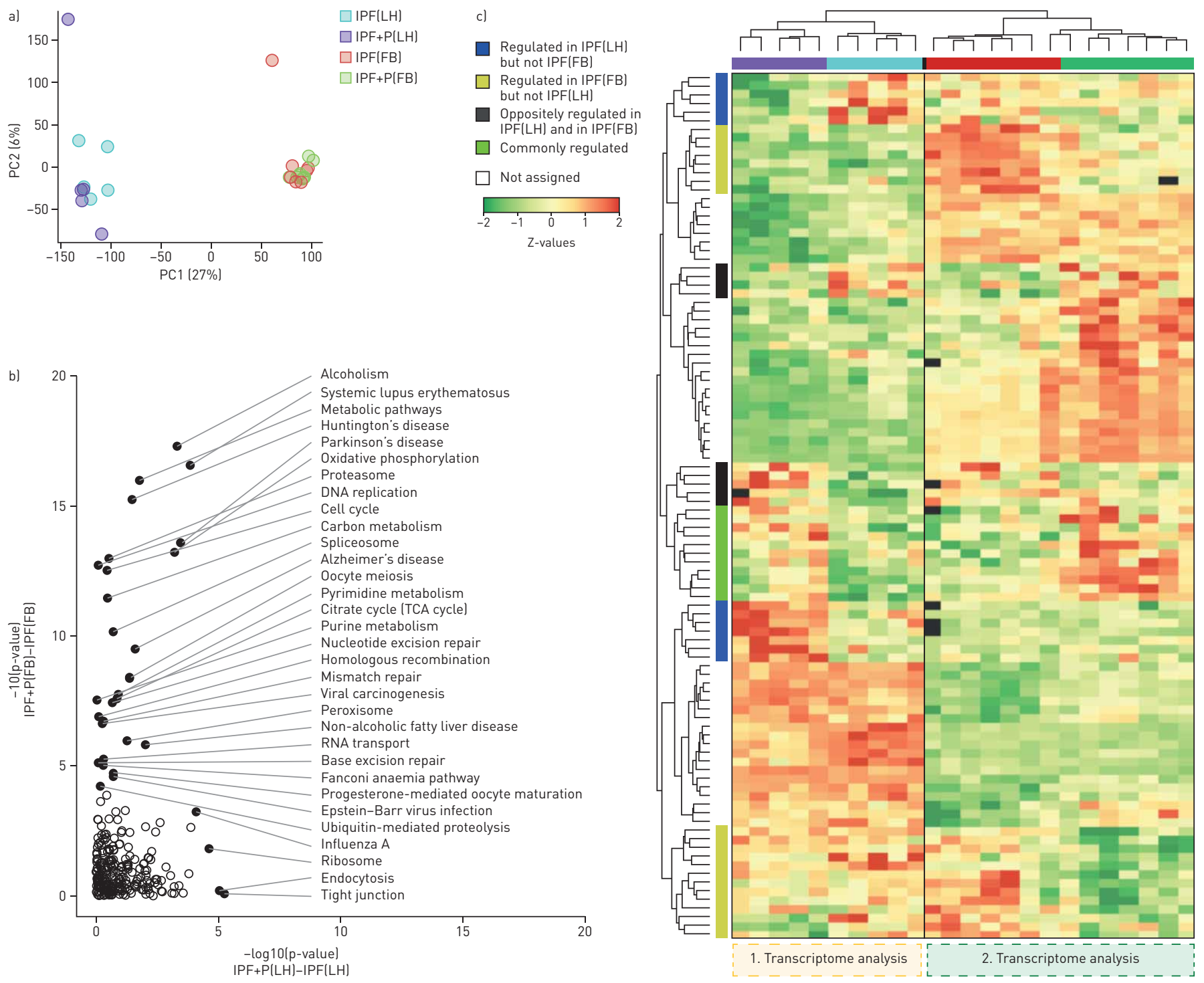

FIGURE 2 Comparison of transcriptomic profiles in human lung homogenates (LH) and human lung fibroblasts (FB) derived from idiopathic pulmonary fibrosis (IPF) patients treated with pirfenidone (P). a) Principal component (PC) analysis of LH and lung FB from pirfenidone-treated and pirfenidone-naïve IPF patients. b) Kyoto Encyclopedia of Gene and Genomes pathway analysis comparing pathway enrichment between FB and LH samples. c) Heat map of significant changes in gene expression with colour-coded grouping (left-hand side) of the top 100 differentially regulated genes between $\mathrm{FB}$ and $\mathrm{LH}$.

nodes. In $\mathrm{LH}$, the selected genes were over-represented in "extracellular matrix" and "immune response" nodes (figure $3 \mathrm{~b}, \mathrm{c}$ ). Due to their strong abundance in our analysis, we first explored the molecular interactions within the ECM and inflammatory response nodes. The interaction network together with parallel expressional annotation of genes regulated in LH and FB once more highlighted differences between LH and FB (figure 3d, e and supplementary figure S2). We also explored representative inflammatory and ECM KEGG pathways and colour-mapped the expressional change after pirfenidone treatment (B- and T-cell receptor signalling and ECM-receptor interaction; supplementary figure S3).

Additionally, the five most downregulated genes in $\mathrm{LH}$ pointed towards a dysbalanced immune system: defensin $\beta$ 4A (DEFB4A), chemokine ligand 6 (CXCL6), serum amyloid A2 (SAA2), serum amyloid A1 (SAA1) and BPI fold containing family A member 1 (BPIFA1) (supplementary table S2). In FB, pirfenidone downregulated genes were involved in transcription: homeobox C9 (HOXC8); cell signalling: sulfatase 2 (SULF2); and osteogenic differentiation: stimulator of chondrogenesis 1 (SCRG1) (supplementary table S3).

Convergently regulated genes in LH and isolated FB due to pirfenidone treatment

Applying a cut-off of LFC> $>1$, we found 803 differentially regulated genes (393 up and 410 down) in $\mathrm{LH}$ (supplementary table S2) and 557 (282 up and 275 down) in FB after pirfenidone treatment in 

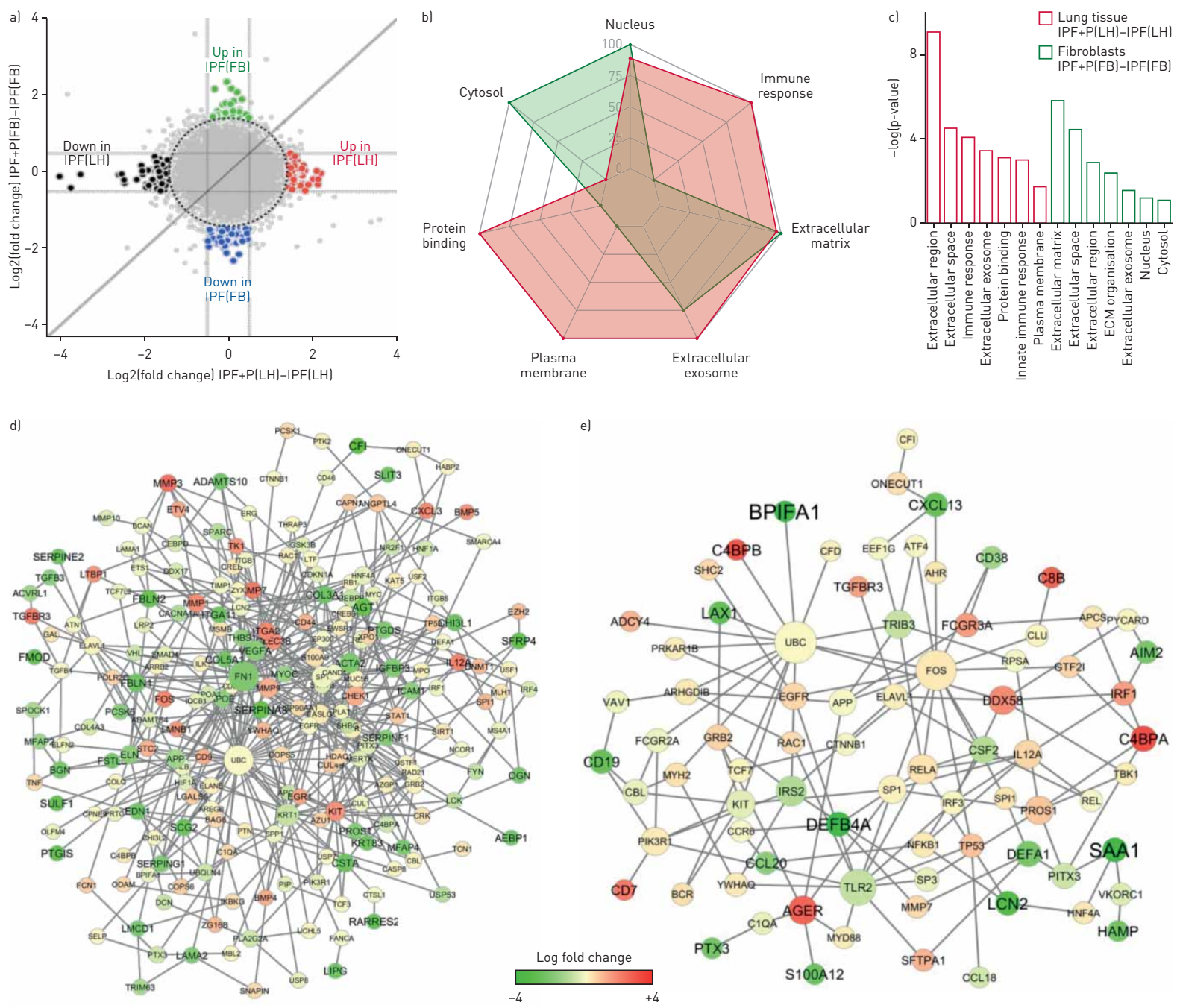

e)
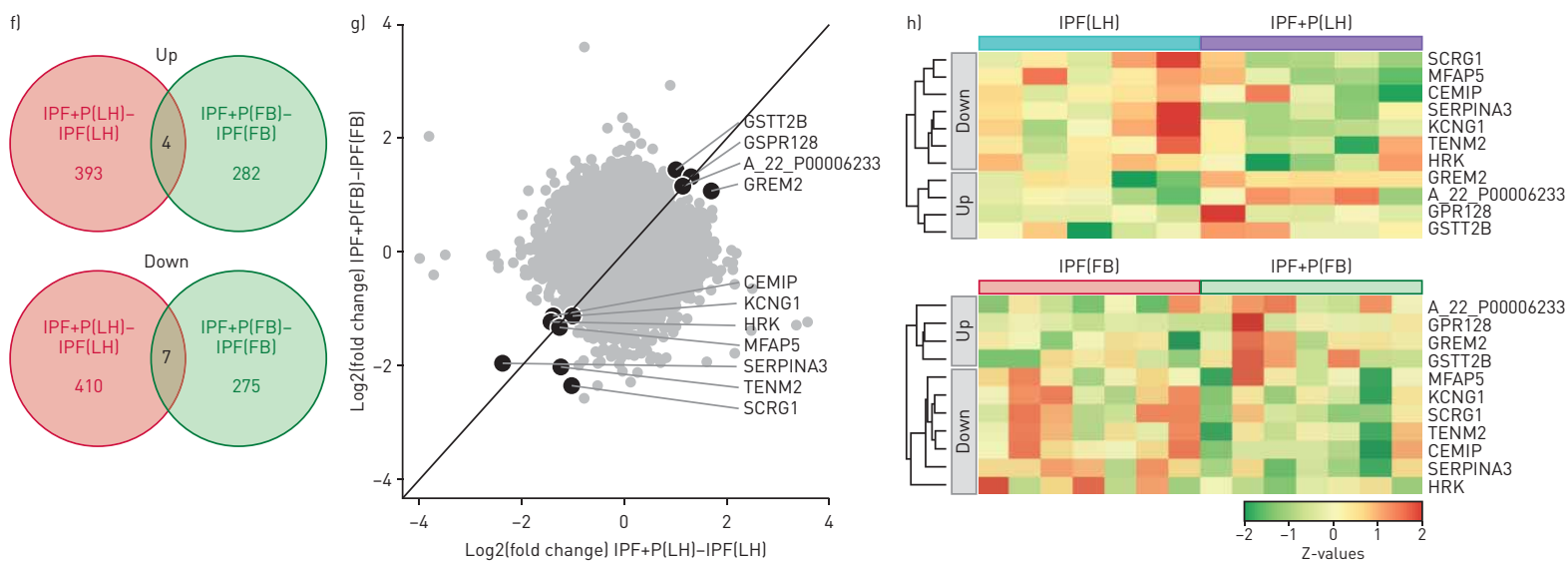

FIGURE 3 Differentially and commonly regulated genes between lung homogenates (LH) and lung fibroblasts (FB) derived from idiopathic pulmonary fibrosis (IPF) patients treated with pirfenidone (P). a) Scatter plot presenting the values of $\log _{2}$ (fold change in expression) for each gene in the IPF (LH) samples (x-axis) versus the IPF(FB) samples (y-axis). Coloured spots represent divergent genes being regulated by pirfenidone only in one of the groups. Genes were considered exclusively regulated in either FB or LH when their point on the scatterplot was outside the dotted circle (radius 1.41 ) and remained within \pm 0.5 for one of the groups (indicated by the horizontal and vertical dotted lines, respectively). b) Spider (radar) chart displays core Gene Ontology (GO) nodes being regulated in IPF(LH) (red) and/or in IPF(FB) (green), and c) shows the top seven single GO nodes significant for each group. $d$, e) Representation of minimum network analysis as performed by NetworkAnalyst showing core protein-protein interactions within the most abundant GO nodes, extracellular matrix (ECM) (d, expression shown for FB) and immune cell response (e, expression shown for LH). f) Venn diagrams representing the number of common up- and downregulated genes between LH and FB from pirfenidone-naïve patients compared to pirfenidone-treated patients (IPF+P). g) Scatterplot presenting the values of log2 (fold change in expression) for each gene regulated upon pirfenidone treatment in the IPF(LH) samples (x-axis) versus the IPF(FB) samples (y-axis). Marked spots represent convergent genes. h) Heat maps representing the individual patient-to-patient variations of commonly regulated genes. 
comparison to the respective controls (supplementary table S3). There were 11 annotated genes with the same direction of regulation (four up and seven down) in both comparison groups (figure $3 \mathrm{f}, \mathrm{g}$ ). The individual patient-to-patient variations of these genes are shown in figure $3 \mathrm{~h}$. The functional involvements of these 11 commonly regulated annotated genes are given in supplementary table S4.

\section{Cell migration-inducing and HA-binding protein as a target of pirfenidone}

Cell migration-inducing and hyaluronan-binding protein (CEMIP) was strongly downregulated by pirfenidone treatment in both our approaches (figure $3 \mathrm{~g}, \mathrm{~h}$ ) and has previously been implicated in several processes relevant to lung fibrosis, namely ECM production, inflammation and cell proliferation (supplementary table S4) [16]. Thus, we explored the role of CEMIP in more detail. The decrease in CEMIP mRNA expression in $\mathrm{IPF}+\mathrm{P}(\mathrm{LH})$ and $\mathrm{IPF}+\mathrm{P}(\mathrm{FB})$ observed in the microarray experiments was confirmed using a validation cohort (figure $4 \mathrm{a}, \mathrm{c})$. Furthermore, IPF $+\mathrm{P}(\mathrm{LH})$ and $\mathrm{IPF}+\mathrm{P}(\mathrm{FB})$ exhibited reduced CEMIP protein expression as compared to $\mathrm{LH}$ and $\mathrm{FB}$ from pirfenidone-naïve IPF patients (figure $4 \mathrm{~b}, \mathrm{~d}$ ). Importantly, both CEMIP mRNA and protein levels were elevated in IPF(LH) and IPF(FB) in comparison to donor samples (figure $4 \mathrm{a}-\mathrm{d}$ ). These results were corroborated by immunohistochemistry, which showed increased staining intensity for CEMIP in the lungs of pirfenidone-naïve IPF patients as compared to donors and pirfenidone-treated patients (figure 4e, f). CEMIP immunoreactivity was mainly observed in alveolar type II cells and (myo)-fibroblasts (as identified by the expression of prosurfactant protein $\mathrm{C}$ and $\alpha$-smooth muscle actin ( $\alpha$-SMA), respectively) in donor and IPF lungs. Positive staining for CEMIP was also observed in endothelial cells (as identified by the expression of von Willebrand factor) in donor lungs (figure $4 \mathrm{f}$ ). These findings indicate that FB are not the exclusive producers of CEMIP in human lungs.

Analysis of circulating CEMIP revealed significantly elevated levels in IPF samples as compared to ageand sex-matched healthy controls (figure $5 \mathrm{a}$ and table 2). Because pirfenidone decreased CEMIP expression in our array analysis, we analysed circulating CEMIP levels in IPF patients before and during pirfenidone treatment. The mean treatment period of these patients was $7.1 \pm 2.5$ months (figure $5 \mathrm{~b}$ ). In six out of seven of the patients, pirfenidone treatment was associated with a marked decrease in CEMIP levels (figure 5c).

\section{CEMIP is involved in invasive properties of IPF FB}

We recently demonstrated that pirfenidone inhibits the Hedgehog (Hh) signalling pathway by targeting GLI proteins [14]. Promoter analysis of the CEMIP gene revealed the presence of the GLI consensus sequence GAACACCCA at the -820 bp position (supplementary figure $4 \mathrm{~A}$ ). In line with this observation, SAG, a synthetic Hh pathway agonist, induced CEMIP protein expression in donor FB. This effect was blocked by pirfenidone and the potent GLI1/2 inhibitor JQ1. Importantly, no additive inhibitory effect was observed when pirfenidone and JQ1 were used simultaneously (supplementary figure 4B), suggesting that pirfenidone itself blocks SAG-triggered CEMIP expression by interfering with GLI transcription factors.

Next we investigated the functional relevance of CEMIP in IPF(FB) via depletion experiments. Knockdown of CEMIP decreased proliferation under basal conditions as well as after stimulation with platelet-derived growth factor-BB or epidermal growth factor (figure 6a), but did not affect apoptosis (figure 6b). Furthermore, silencing of CEMIP inhibited migration and increased the time for wound closure (figure $6 c, d$ ). In addition, knockdown impaired stress fibre formation (figure $6 \mathrm{e}$ ) and reduced expression of collagen I but did not affect the expression of fibronectin, matrix metalloprotease-2 or $\alpha$-SMA (figure $6 \mathrm{f}-\mathrm{h}$ ). mRNA expression of the senescence markers p21 and p53 was downregulated following CEMIP depletion; however, no changes were apparent at the protein level (figure $6 f-h$ ). Given that CEMIP is implicated in the catabolism of HA, we examined whether pirfenidone treatment affects deposition of HA in the lungs of IPF patients. As depicted in figure 6i, j, more prominent accumulation of HA was observed in pirfenidone-treated than in pirfenidone-naïe IPF patients. Furthermore, our microarray analysis revealed that pirfenidone differently regulated the HA-mediated motility receptor (HMMR) and HA and proteoglycan link protein 4 (HAPLN4) in both IPF(LH) and IPF(FB). Additionally, pirfenidone significantly affected the expression of HA synthase 1 (HAS1) and HA-binding protein 2 (HABP2) specifically in IPF(LH), while there was altered expression of inter- $\alpha$-trypsin inhibitor heavy chain 3 (ITIH3), a HA-binding protein essential for ECM stabilisation, and the CD44 molecule in IPF(FB) only (supplementary tables S5 and S6).

\section{Effects of pirfenidone in vitro}

In the final set of experiments, we extended our transcriptomic analysis to genes that were dysregulated in $\mathrm{IPF}(\mathrm{FB})$, kept in culture for several passages, and then treated with pirfenidone in vitro. The rationale behind this was 1) to have a complementary in vitro experimental setting of pirfenidone action and 2) to single out the specific signalling mechanisms of pirfenidone in FB without the effects of the global response to 

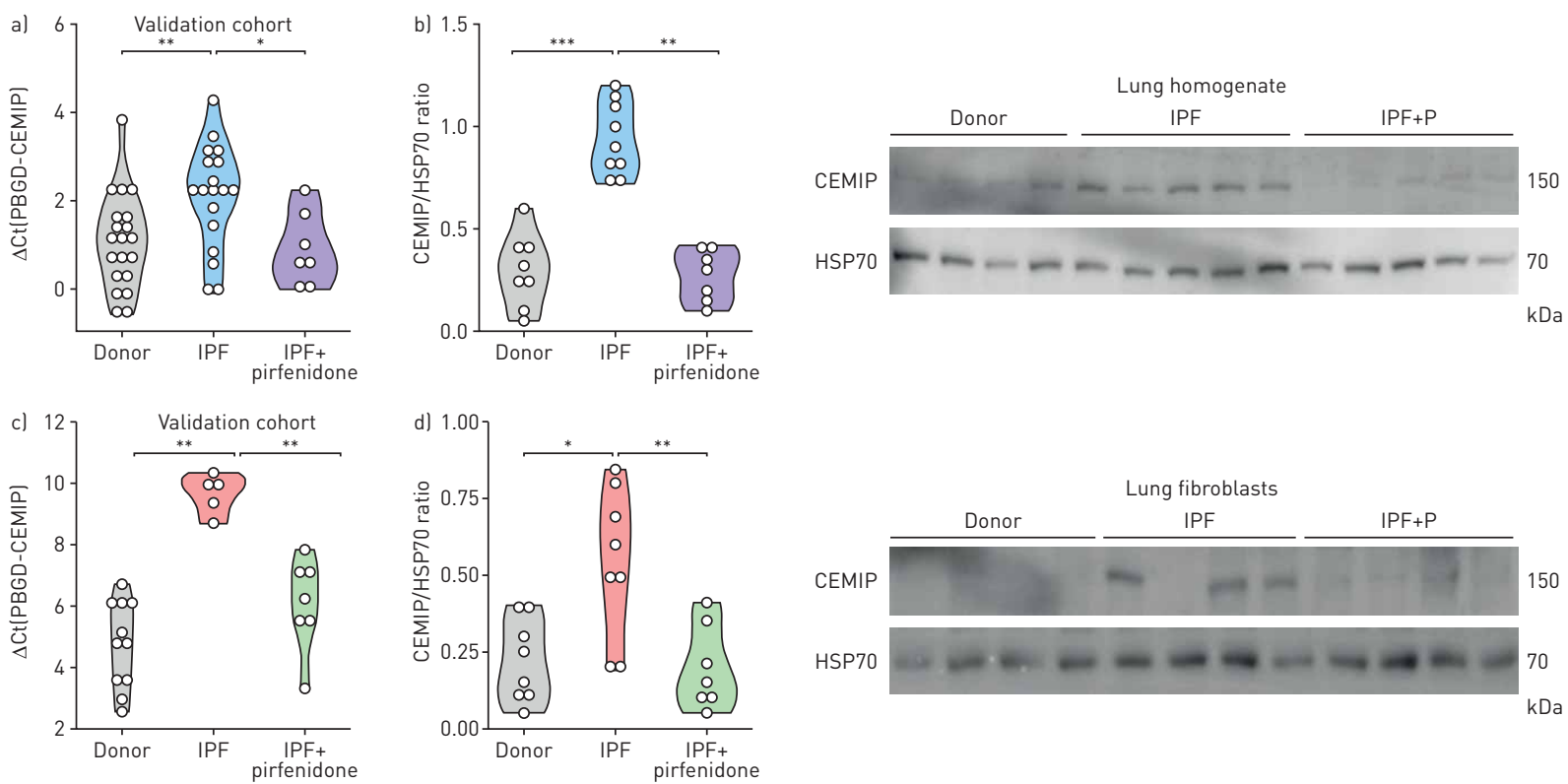

e)
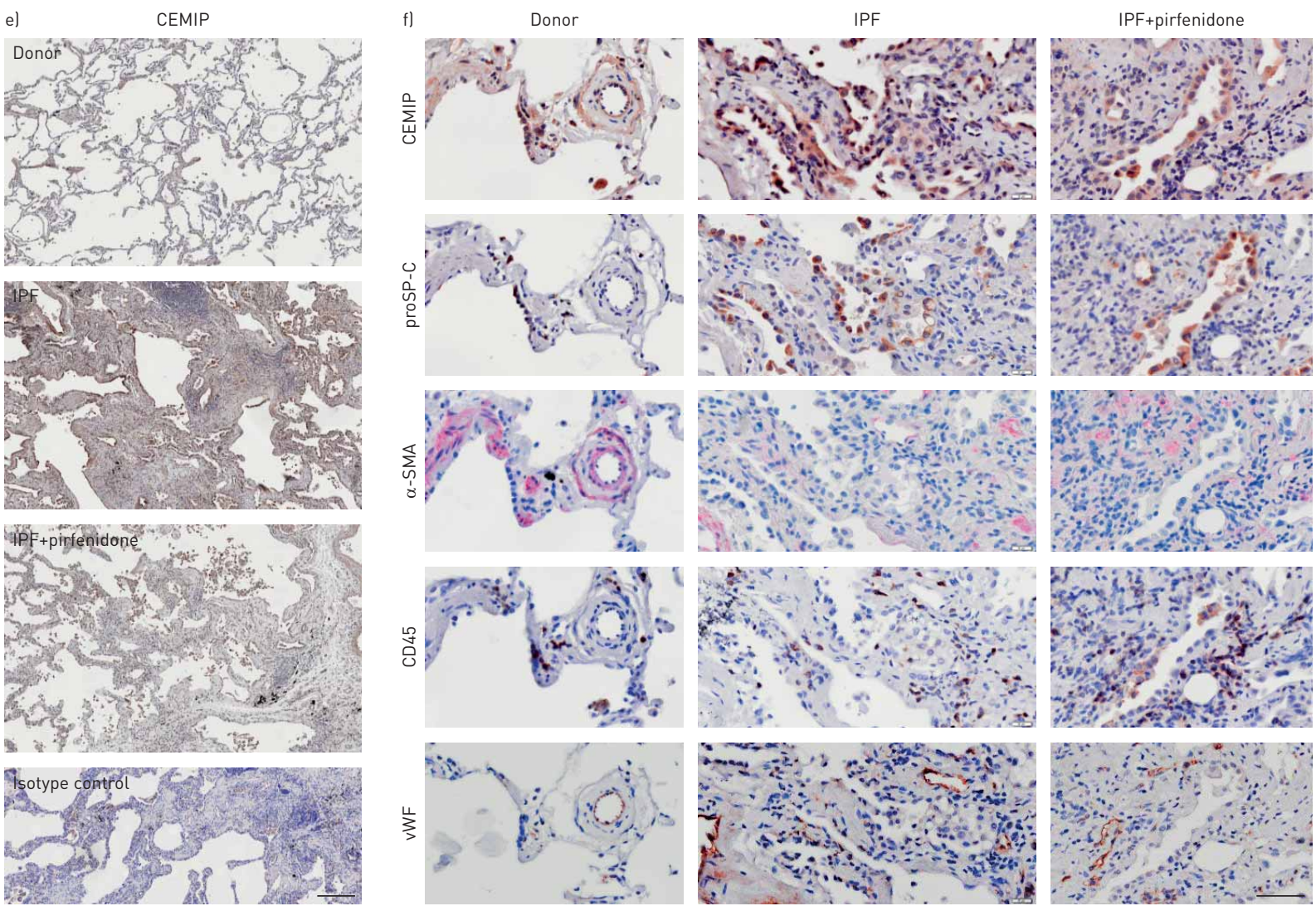

FIGURE 4 CEMIP regulation in lung fibrosis upon pirfenidone (P) treatment. a) CEMIP mRNA expression in lung homogenates (LH) of an independent validation cohort ( $n=20$ for donor; $n=18$ for idiopathic pulmonary fibrosis (IPF); $n=7$ for IPF+P). b) CEMIP protein levels in LH of donors and pirfenidone-naïve and pirfenidone-treated IPF patients ( $n=8$ for donor; $n=9$ for IPF; $n=7$ for IPF+P). c) CEMIP mRNA expression in lung fibroblasts (FB) isolated from donor lungs and pirfenidone-naïve and pirfenidone-treated IPF patients of an independent validation cohort ( $\mathrm{n}=11$ for donor; $n=5$ for IPF; $n=7$ for IPF+P). d) CEMIP protein levels in FB isolated from lungs of donors and pirfenidone-naïve and pirfenidone-treated IPF patients ( $n=8$ for donor; $n=8$ for IPF; $n=7$ for IPF+P). mRNA levels were assessed by quantitative PCR, proteins levels by Western blotting. $P B G D$ was used as a reference gene in quantitative PCR and HSP70 as a loading control in Western blotting. Biological replicates are shown. For statistical analysis, one-way ANOVA with Tukey's multiple comparisons test was used. ${ }^{*} p<0.05,{ }^{* *} p<0.01 .{ }^{* * *} p<0.001$. e) Overview images of CEMIP immunoreactivity in lungs of donors and pirfenidone-naïve and pirfenidone-treated IPF patients. Isotype control antibody was used to confirm the specificity of the staining; scale bar $=200 \mu \mathrm{m}$. f) High magnification images of CEMIP, prosurfactant protein C (proSP-C), $\alpha$-smooth muscle actin ( $\alpha$-SMA), CD45 and von Willebrand factor (vWF) immunostainings on serial sections of lungs from donors and pirfenidone-naïve and pirfenidone-treated IPF patients; scale bar=50 $\mu \mathrm{m}$. 


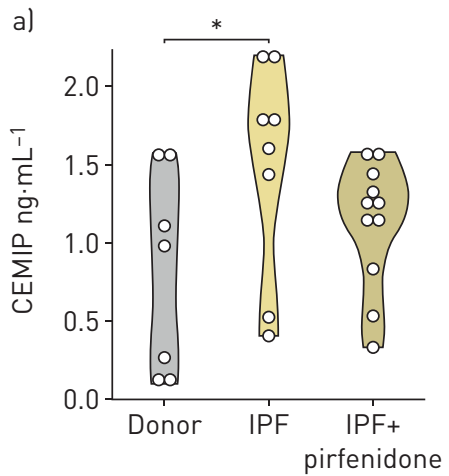

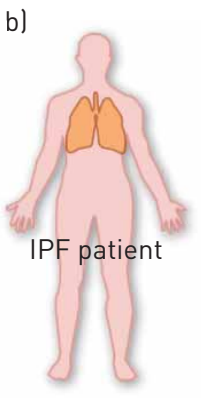

Pre-treatment blood draw

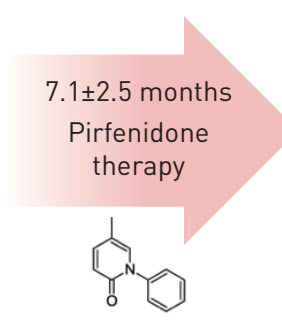

Post-treatment blood draw

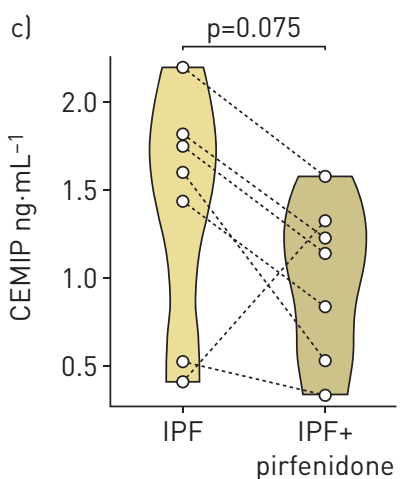

FIGURE 5 CEMIP levels in the plasma of healthy controls and pirfenidone-naive and pirfenidone-treated idiopathic pulmonary fibrosis (IPF) patients. a) CEMIP plasma levels of healthy control subjects and pirfenidone-naïve and pirfenidone-treated IPF patients. Kruskal-Wallis test with Dunn's multiple comparison test; ${ }^{*} p<0.05$. b) Schematic of follow-up in IPF patients before and during pirfenidone treatment. c) CEMIP plasma levels at baseline and after $7.1 \pm 2.5$ months of pirfenidone treatment. Wilcoxon matched pairs test.

pirfenidone when FB are in their natural microenvironment. To this end, we performed a comparative study between 1) $\mathrm{FB}$ isolated from pirfenidone-treated (IPF+P(FB)) versus pirfenidone-naïve (IPF(FB)) patients and 2) lung $\mathrm{FB}$ isolated from IPF patients, cultured and exposed to pirfenidone in vitro (IPF( $\mathrm{FB}+\mathrm{P}$ ) (figure 7a). Applying a $L F C>|1|$, we found a total of 743 genes that were regulated in in vitro pirfenidone-treated FB (supplementary table S7). Hierarchical clustering of the top 100 regulated genes showed complete separation of the transcription profiles from $\mathrm{FB}$, treated both in vivo as well as in vitro (figure $7 \mathrm{~b}$ ). Of note, we found that gene expression varied considerably between FB from in vivo and in vitro settings, underlining the influence of in vitro culturing on FB. Nevertheless, a comparison of pirfenidone-regulated genes from $\mathrm{IPF}(\mathrm{FB}+\mathrm{P})$ and $\mathrm{IPF}+\mathrm{P}(\mathrm{FB})$ revealed 23 genes with the same expression pattern (17 up and six down; figure 7c, $\mathrm{d}$ and supplementary table S8). Among the downregulated genes were endothelin 1 (EDN1) and 5-hydroxytryptamine receptor 2B (HTR2B), which are both part of the $\mathrm{G}$ protein-coupled receptor signal transduction pathways. The upregulated genes were annotated to the following biological processes: transforming growth factor- $\beta$ (TGF- $\beta$ ) receptor signalling pathway, transcription from RNA polymerase II promoter and cellular lipid metabolic process. The heat maps in figure 7e represent the individual patient-to-patient variations of commonly regulated genes in both settings.

\section{Discussion}

The clinical success of pirfenidone in the treatment of IPF is attributed to its pleiotropic mode of action. Numerous in vitro and in vivo studies have demonstrated that pirfenidone exhibits anti-fibrotic, anti-inflammatory and anti-oxidant effects [15]; however, it remains unclear which of these effects occur at the therapeutic doses achieved in humans.

\begin{tabular}{|c|c|c|c|}
\hline Variable & Healthy & IPF baseline & IPF+P \\
\hline CEMIP plasma levels $\mathrm{ng} \cdot \mathrm{mL}^{-1}$ & $0.83 \pm 0.6$ & $1.49 \pm 0.7$ & $1.13 \pm 0.4$ \\
\hline Subjects & 7 & 8 & 11 \\
\hline Age years & $58.5 \pm 6.6$ & $59.4 \pm 12.7$ & $61.6 \pm 10.8$ \\
\hline \multicolumn{4}{|l|}{ Sex } \\
\hline Male & 5 & 5 & 9 \\
\hline Female & 2 & 3 & 2 \\
\hline \multicolumn{4}{|l|}{ Smoking status } \\
\hline Never & 2 & 1 & 2 \\
\hline Former & 2 & 7 & 9 \\
\hline Current & 3 & 0 & 0 \\
\hline FVC \% pred & & $60.1 \pm 12.5$ & $61.1 \pm 9.9$ \\
\hline Histological confirmation of a UIP pattern \% & & 100 & 100 \\
\hline Daily dose of pirfenidone $g$ & & & $2.4 \pm 0.0$ \\
\hline Duration of pirfenidone treatment months & & & $7.1 \pm 2.5$ \\
\hline
\end{tabular}

Data presented as $\mathrm{n}$ or mean $\pm \mathrm{SD}$, unless otherwise indicated. IPF: idiopathic pulmonary fibrosis; P: pirfenidone; FVC: forced vital capacity; UIP: usual interstitial pneumonia. 

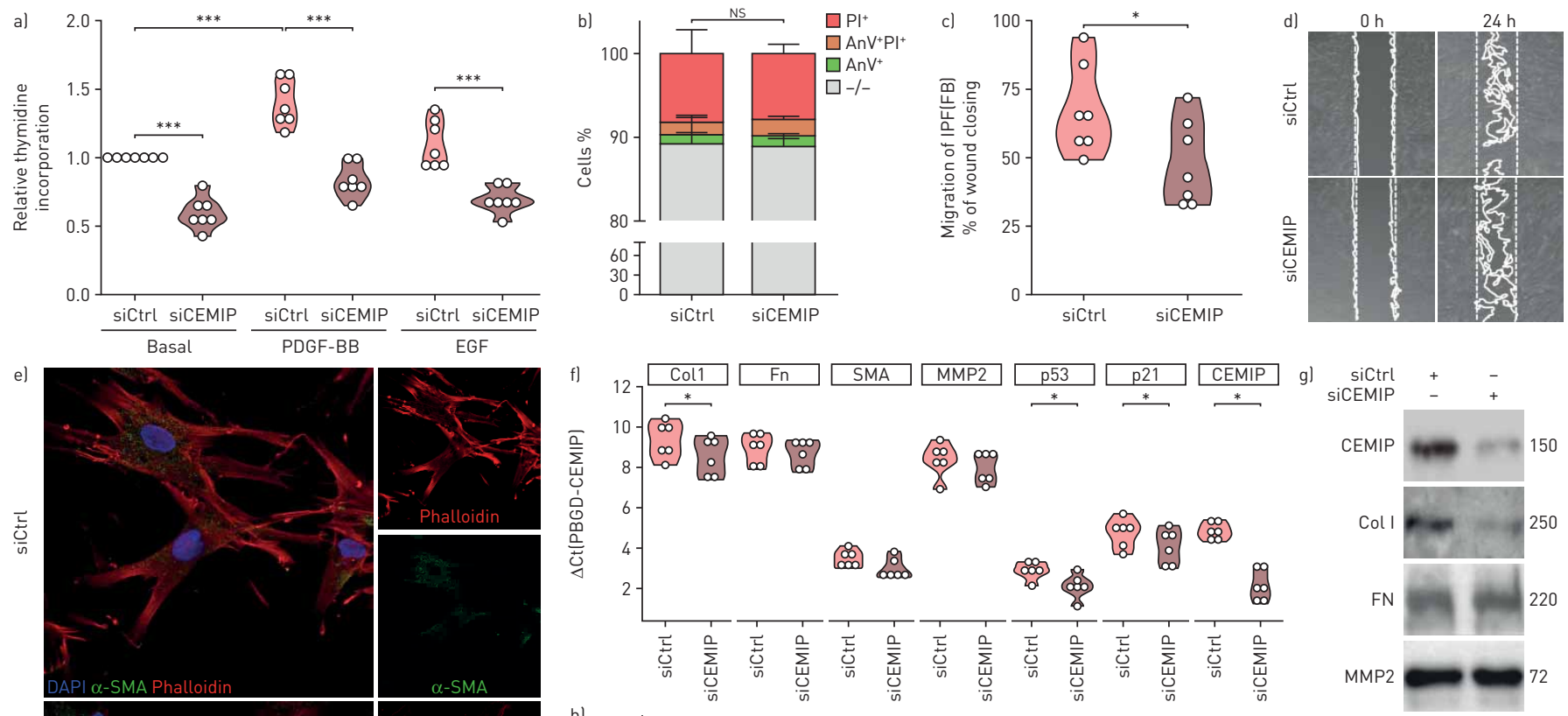

\section{\begin{tabular}{ll}
\hline MMP2 \\
\hline$\infty$ \\
$\infty$ \\
$\infty$ & 009 \\
$\infty$ & 0
\end{tabular}}
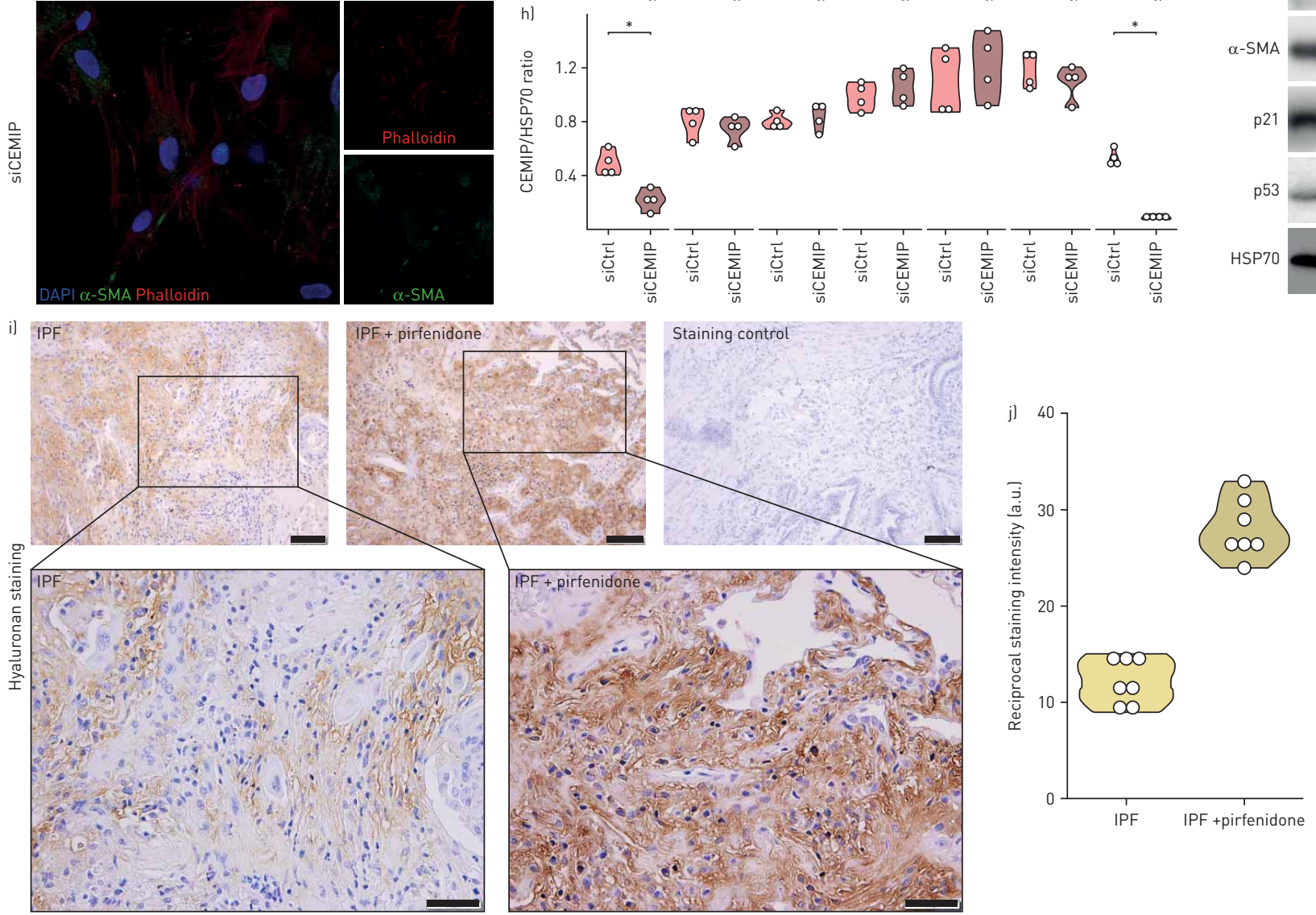

FIGURE 6 Effects of knockdown of CEMIP on idiopathic pulmonary fibrosis (IPF) pulmonary fibroblasts (FB). a) Proliferation of IPF(FB) treated with CEMIP siRNA (siCEMIP) or non-targeting control siRNA (siCtrl) at basal conditions and upon stimulation with platelet-derived growth factor (PDGF-BB) or epidermal growth factor (EGF). b) Apoptosis of IPF(FB) measured by AnnexinV-FITC (AnV) and propidium iodide (PI) staining. A minimum of 5000 cells was measured. c, d) Quantification (c) and representative pictures (d) of IPF(FB) migration after $24 \mathrm{~h}$ of siCEMIP or siCtrl treatment. e) $\alpha$-smooth muscle actin ( $\alpha$-SMA) (green) and phalloidin (red) fluorescence staining of IPF(FB) after treatment with siCEMIP or siCtrl. In a-e the combined data of two independent experiments with $n=4$ biological replicates are presented. $f$ ) mRNA and g) protein levels of markers for extracellular matrix (ECM) production/turnover (collagen I (Col1), fibronectin (FN), matrix metalloprotease-2 (MMP2)), FB differentiation $(\alpha-S M A)$ and FB senescence (p53, p21). mRNA levels were assessed by quantitative PCR $(n=6)$ and protein levels by Western blotting ( $n=4)$. $P B G D$ was used as a reference gene in quantitative PCR and HSP70 as a loading control in Western blotting. h) Quantification of g. For statistical analysis a Mann-Whitney test was used. ${ }^{*}: p<0.05 ;{ }^{* *}: p<0.01 ;{ }^{* * *}: p<0.001 . i, j$ ) Affinity-histochemical staining (i) and quantification (j) of hyaluronan on IPF lungs of pirfenidone-naïve and pirfenidone-treated patients. Upper panel scale bar=100 $\mu \mathrm{m}$, inset/lower panel scale bar=50 $\mu \mathrm{m}$. Sections pre-treated with hyaluronidase were used as a staining control. 
a)

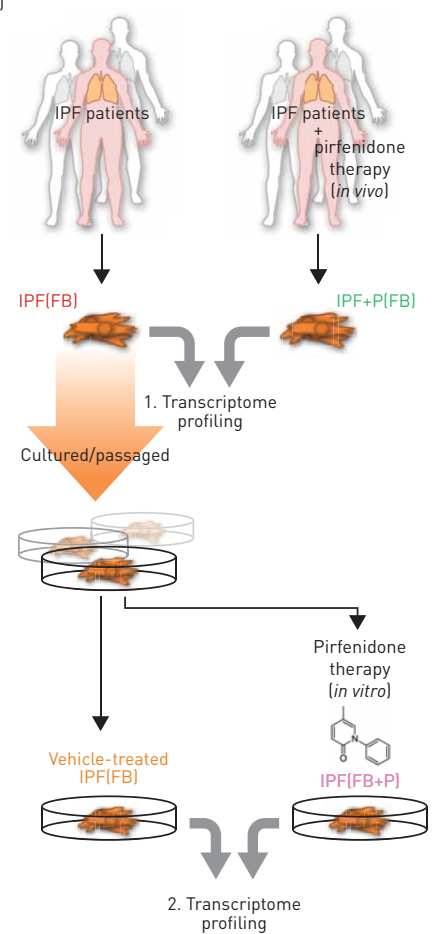

c)
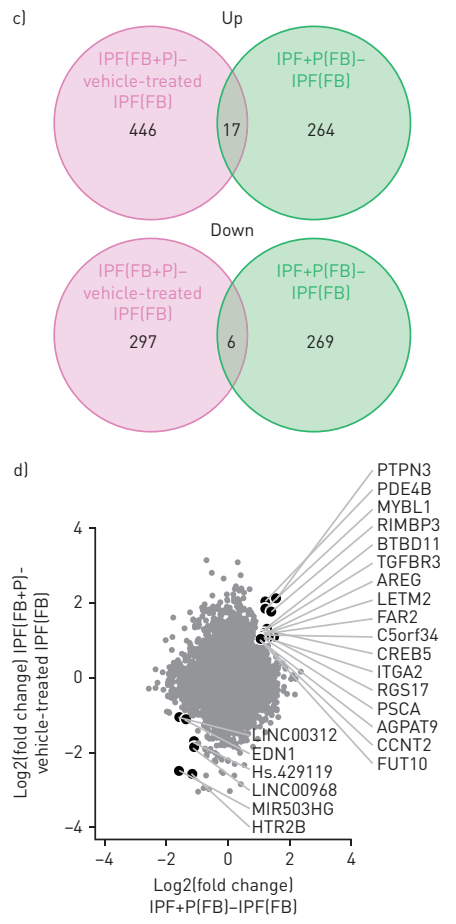

b)

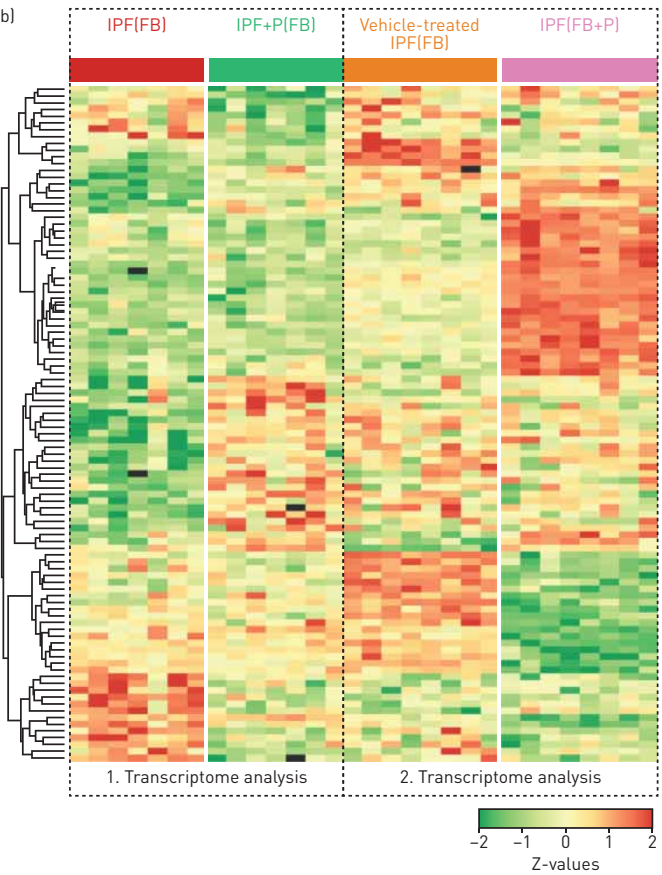

e)
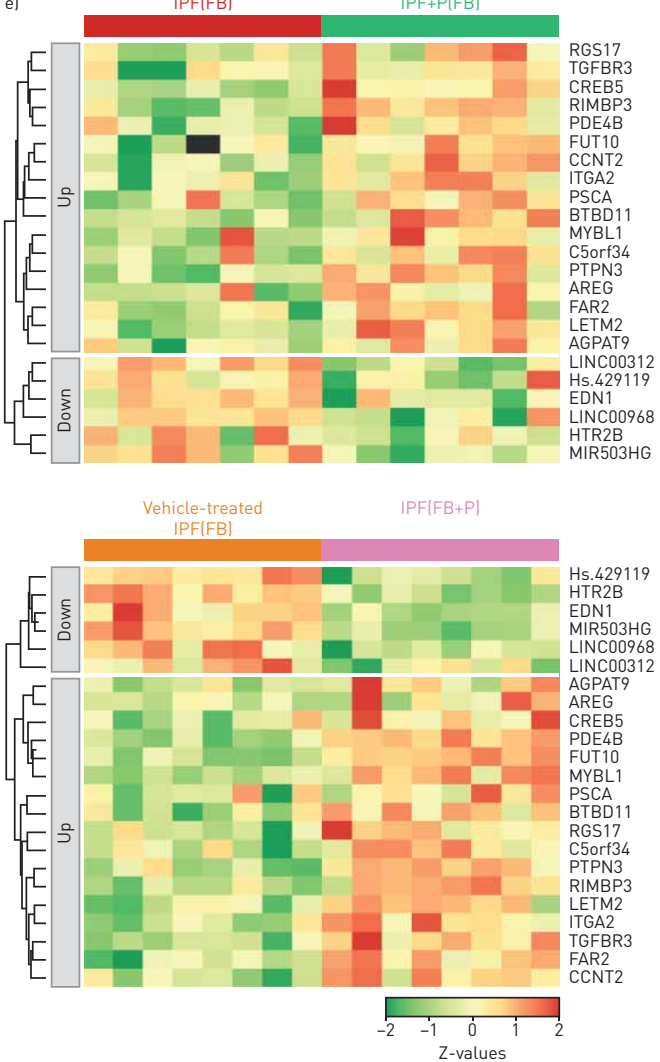

FIGURE 7 Transcriptomic profiling of human lung fibroblasts (FB) treated with pirfenidone (P) in vitro. a) Schematic overview of the experimental design. FB used for the first transcriptomic profiling (in situl were used immediately after isolation (passage one). In the in vitro settings, FB were used between passage three to five (second transcriptomic profiling). b) Heat map representation of significant changes in gene expression between in situ (idiopathic pulmonary fibrosis (IPF)+P(FB)) and in vitro (IPF(FB+P)) treated $F B$. c) Venn diagrams showing the number of common up- and downregulated genes between IPF+P(FB) and IPF(FB+P). d) Scatterplot presenting the values of log2 (fold change in expression) for each gene in the IPF+P(FB) samples (x-axis) versus the in vitro treated FB (IPF(FB+P)) (y-axis). Marked spots represent genes with an absolute log 2 fold change of $>1$ in both experimental approaches (in situ and in vitro). e) Heat maps representing the individual patient-to-patient variations of commonly regulated genes. 
In the present study, we performed gene expression profiling analysis to identify pirfenidone's mode of action. The analysis was performed on multiple levels using LH samples and freshly isolated FB derived from IPF patients who were or were not treated with pirfenidone. This in situ approach was further corroborated by our in vitro study, in which fibroblasts isolated from IPF patients were exposed to pirfenidone in cell culture. Pirfenidone treatment was associated with major changes in inflammatory processes and cell-cell contacts in LH, while in FB the most significantly perturbed pathways were related to metabolic reprogramming, growth and cell division. Genes regulated in both specimens primarily belonged to the ECM.

Interactions between ECM molecules and inflammatory cells/mediators ensure a proper response of the lung to insults, and their dysregulation can lead to an aberrant damage response and, finally, fibrosis. The mutual relationship between ECM-producing FB and different subpopulations of inflammatory cells in lung fibrosis is supported by numerous studies. For instance, degradation products of HA have been found to stimulate B-cells to produce various pro-fibrogenic cytokines, including potent activators of $\mathrm{FB}$ such as TGF- $\beta 1$, interleukin (IL)- 6 and IL-4 [17]. Interestingly, abnormal B- and T-cell aggregates have been shown in IPF lungs and diverse IgG autoantibodies have been reported in IPF plasma [2, 18-20].

The close interplay between the ECM and immune responses is supported by our data, demonstrating that among the genes significantly downregulated by pirfenidone in the LH were those involved in regulating innate and adaptive immunity, including CXCL6 and tumour necrosis factor receptor superfamily member 17 (TNFRSF17). Strikingly, TNFRSF17 has been shown to control the development of B-cells and thereby the autoimmune responses [21]. In pulmonary FB, pirfenidone therapy mainly suppressed the expression of SULF2, an enzyme involved in post-translational modification of ECM components [22]. Increased sulfation of ECM heparan sulfate proteoglycans has already been described in the lungs of IPF patients, suggesting altered structural and growth factor binding capacity of the fibrotic matrix [23].

In both pulmonary FB and LH derived from pirfenidone-treated patients, one of the most upregulated genes was gremlin 2 (GREM2), while one of the most significantly downregulated genes was CEMIP. The role of gremlin in fibrogenesis remains controversial, with studies demonstrating its pro- and anti-fibrotic activities [24, 25]. Anti-fibrotic properties of gremlin are associated with its ability to upregulate fibroblast growth factor 10 (FGF10) and thus facilitate the repair of injured alveolar epithelium [26, 27]. The contribution of CEMIP to the pathogenesis of IPF has not been acknowledged to date; thus, our study is the first to demonstrate its potent pro-fibrotic actions.

CEMIP influences the extracellular environment by participating in the catabolism of HA [28], which not only alters the strength, lubrication and hydration of ECM but also regulates adhesion, migration, proliferation and differentiation of a variety of cells [29]. We found a marked increase in CEMIP mRNA and protein expression in LH and FB of IPF patients as compared to donors. Most importantly, CEMIP expression was suppressed by pirfenidone treatment in both the primary and the validation cohort of our IPF patients. Furthermore, pirfenidone therapy resulted in a sharp decrease of CEMIP plasma levels in IPF patients who had high CEMIP plasma levels at baseline. These findings strongly encourage further investigations and suggest that CEMIP could be used as a predictive biomarker to identify IPF patients with profound alterations in ECM architecture and inflammation who are most likely to respond favourably to this treatment.

Although in vivo studies are needed to delineate the contribution of CEMIP to the development of lung fibrosis, our results suggest that CEMIP depletion suppresses the proliferation of IPF lung FB in response to different pro-fibrotic stimuli, impairs migration of these cells, and lowers collagen I production. Furthermore, the decreased CEMIP expression may stabilise HA fibres, as suggested from our staining procedures. HA can differentially promote or suppress fibrosis depending on the length of its carbohydrate chain. In the lungs of bleomycin-treated mice, low-molecular-weight HA exerts potent pro-inflammatory effects and exacerbates inflammatory responses, which consequently lead to the progression of lung fibrosis [30, 31]. By contrast, high-molecular-weight HA, which is mainly produced by HAS1 and HAS2, is crucial for regenerative tissue repair. In the skin, an IL-10-triggered increase in HAS1 and HAS2 expression and decrease in hyaluronidase (HYAL) 1, HYAL2 and CEMIP expression reduces scar formation in different wound models [32]. In the lung, depletion of HAS2 in alveolar type II cells (ATIIC) impairs the renewal capacity of ATIIC and exacerbates lung fibrosis upon bleomycin instillation [33]. Thus, our data support an important role for an HA-rich wound ECM for proper tissue regeneration and suggest CEMIP as a potential therapeutic target in diseases in which dysregulated inflammation and HA intersect $[23,29,34]$.

The direct comparison of gene profile changes of IPF pulmonary FB upon in vitro treatment with pirfenidone with pulmonary FB isolated from pirfenidone-treated IPF patients revealed only 23 genes with a matching expression change. Among these, only two protein-coding genes, EDN1 and 5-HTR2B, were 
downregulated. EDN1 and 5-HTR2B are involved in the pathogenesis of IPF; EDN1 through the induction of fibroblast proliferation and transdifferentiation [35] and 5-HTR2B via the regulation of TGF- $\beta 1$-triggered collagen production [36]. Although the contribution of EDN1 and 5-HTR2B to the development of lung fibrosis has been examined in experimental models of lung fibrosis, the role of these molecules in the pathogenesis of human IPF is still unclear.

Even though valuable insights can be gained from in vitro experiments on the direct effects of pirfenidone, our results demonstrate that these experiments do not provide a full picture of the biological complexity of pirfenidone action in IPF and should be regarded with caution. In vitro results can be influenced by culture conditions, cell passage and direct exposure of a single cell type to a pharmacological compound at non-physiological levels. Our previous findings demonstrated that pirfenidone inhibits pro-fibrotic activities of cultured lung FB only when it is used in a concentration strongly exceeding the levels observed in IPF plasma [14]. We cannot exclude the possibility that the high pirfenidone concentrations in vitro could lead to additional off-target effects, explaining the small overlap between IPF+P(FB) and IPF(FB+P) groups. Thus, in vitro studies might be used to generate a hypothesis, which then has to be tested using in situ and in vivo approaches.

The main limitation of this study was that our lung samples do not represent a random sample from a prospective randomised controlled study in which pirfenidone was compared to placebo. In fact, we used tissue samples from explanted end-stage lungs of IPF patients who were or were not treated with pirfenidone. Therefore, it is possible that this patient selection may have biased the results. Although all patients had end-stage lung disease, we found highly significant differences associated with pirfenidone treatment. Further, the low number of patients used for the analysis could limit the reliability of the results; however, two independent approaches increased the robustness of the results. By combining the analysis of biomaterial from pirfenidone-treated and pirfenidone-naïve IPF patients and that of isolated lung FB that were treated with pirfenidone in vitro, we were able to identify a consistent pattern of pirfenidone-induced changes in the gene expression profiles.

Although we are far away from fully understanding the pathogenesis of IPF and the effects of pirfenidone, our results point to an important role for innate and adaptive immune responses as well as ECM organisation in the progressive and irreversible lung tissue scarring. Our approach provides a basis for new combination-based therapeutic strategies improving the effectiveness of pirfenidone in IPF.

Conflict of interest: A. Olschewski reports grants and honoraria for speaking from Pfizer, outside the submitted work. W. Seeger reports personal fees from Pfizer, Novartis, United Therapeutics, Actelion, Vectura, Savara, Medspray and Bayer AG, outside the submitted work. H. Olschewski reports grants from Intermune/Roche, and grants and personal fees from Boehringer, outside the submitted work. All other authors have nothing to disclose.

Support statement: Funding for this study was received from the German Research Foundation (WY119/1-3 to M. Wygrecka), the Else Kröner-Fresenius-Foundation (to M. Wygrecka), the Excellence Cluster "Cardiopulmonary System" (to M. Wygrecka), the German Center for Lung Research (to M. Wygrecka), the University Medical Center Giessen and Marburg (to M. Wygrecka), the Austrian Science Fund (P27848-B28 to G. Kwapiszewska), the Jubilee Foundation of the Austrian National Bank (16187 to G. Kwapiszewska) and the Austrian Research Promotion Agency (858308 to G. Kwapiszewska and H. Thekkekara Puthenparampil). Funding information for this article has been deposited with the Crossref Funder Registry.

\section{References}

1 Collard HR, Ryerson CJ, Corte TJ, et al. Acute exacerbation of idiopathic pulmonary fibrosis. An International Working Group Report. Am J Respir Crit Care Med 2016; 194: 265-275.

2 Martinez FJ, Collard HR, Pardo A, et al. Idiopathic pulmonary fibrosis. Nat Rev Dis Primers 2017; 3: 17074.

3 Raghu G, Brown KK, Bradford WZ, et al. A placebo-controlled trial of interferon gamma-1b in patients with idiopathic pulmonary fibrosis. N Engl J Med 2004; 350: 125-133.

4 Daniels CE, Lasky JA, Limper AH, et al. Imatinib treatment for idiopathic pulmonary fibrosis: randomized placebo-controlled trial results. Am J Respir Crit Care Med 2010; 181: 604-610.

5 King TE Jr, Behr J, Brown KK, et al. BUILD-1: a randomized placebo-controlled trial of bosentan in idiopathic pulmonary fibrosis. Am J Respir Crit Care Med 2008; 177: 75-81.

6 Raghu G, Behr J, Brown KK, et al. Treatment of idiopathic pulmonary fibrosis with ambrisentan: a parallel, randomized trial. Ann Intern Med 2013; 158: 641-649.

7 Raghu G, Rochwerg B, Zhang Y, et al. An Official ATS/ERS/JRS/ALAT Clinical Practice Guideline: treatment of idiopathic pulmonary fibrosis. An update of the 2011 Clinical Practice Guideline. Am J Respir Crit Care Med 2015; 192: e3-e19.

8 King TE Jr., Bradford WZ, Castro-Bernardini S, et al. A phase 3 trial of pirfenidone in patients with idiopathic pulmonary fibrosis. N Engl J Med 2014; 370: 2083-2092.

9 Noble PW, Albera C, Bradford WZ, et al. Pirfenidone in patients with idiopathic pulmonary fibrosis (CAPACITY): two randomised trials. Lancet 2011; 377: 1760-1769.

10 Taniguchi H, Ebina M, Kondoh Y, et al. Pirfenidone in idiopathic pulmonary fibrosis. Eur Respir J 2010; 35: $821-829$. 
11 Azuma A, Nukiwa T, Tsuboi E, et al. Double-blind, placebo-controlled trial of pirfenidone in patients with idiopathic pulmonary fibrosis. Am J Respir Crit Care Med 2005; 171: 1040-1047.

12 Noble PW, Albera C, Bradford WZ, et al. Pirfenidone for idiopathic pulmonary fibrosis: analysis of pooled data from three multinational phase 3 trials. Eur Respir J 2016; 47: 243-253.

13 Chaudhuri N, Duck A, Frank R, et al. Real world experiences: pirfenidone is well tolerated in patients with idiopathic pulmonary fibrosis. Respir Med 2014; 108: 224-226.

14 Didiasova M, Singh R, Wilhelm J, et al. Pirfenidone exerts antifibrotic effects through inhibition of GLI transcription factors. FASEB J 2017; 31: 1916-1928.

15 Lopez-de la Mora DA, Sanchez-Roque C, Montoya-Buelna M, et al. Role and new insights of pirfenidone in fibrotic diseases. Int J Med Sci 2015; 12: 840-847.

16 Kohi S, Sato N, Koga A, et al. KIAA1199 is induced by inflammation and enhances malignant phenotype in pancreatic cancer. Oncotarget 2017; 8: 17156-17163.

17 Yoshizaki A, Iwata Y, Komura K, et al. CD19 regulates skin and lung fibrosis via Toll-like receptor signaling in a model of bleomycin-induced scleroderma. Am J Pathol 2008; 172: 1650-1663.

18 Marchal-Somme J, Uzunhan Y, Marchand-Adam S, et al. Cutting edge: nonproliferating mature immune cells form a novel type of organized lymphoid structure in idiopathic pulmonary fibrosis. J Immunol 2006; 176: $5735-5739$.

19 Xue J, Kass DJ, Bon J, et al. Plasma B lymphocyte stimulator and B cell differentiation in idiopathic pulmonary fibrosis patients. J Immunol 2013; 191: 2089-2095.

20 Taille C, Grootenboer-Mignot S, Boursier C, et al. Identification of periplakin as a new target for autoreactivity in idiopathic pulmonary fibrosis. Am J Respir Crit Care Med 2011; 183: 759-766.

21 Rickert RC, Jellusova J, Miletic AV. Signaling by the tumor necrosis factor receptor superfamily in B-cell biology and disease. Immunol Rev 2011; 244: 115-133.

22 Rosen SD, Lemjabbar-Alaoui H. Sulf-2: an extracellular modulator of cell signaling and a cancer target candidate. Expert Opin Ther Targets 2010; 14: 935-949.

23 Westergren-Thorsson G, Hedstrom U, Nybom A, et al. Increased deposition of glycosaminoglycans and altered structure of heparan sulfate in idiopathic pulmonary fibrosis. Int J Biochem Cell Biol 2017; 83: 27-38.

24 Koli K, Myllarniemi M, Vuorinen K, et al. Bone morphogenetic protein-4 inhibitor gremlin is overexpressed in idiopathic pulmonary fibrosis. Am J Pathol 2006; 169: 61-71.

25 Myllarniemi M, Lindholm P, Ryynanen MJ, et al. Gremlin-mediated decrease in bone morphogenetic protein signaling promotes pulmonary fibrosis. Am J Respir Crit Care Med 2008; 177: 321-329.

26 Farkas L, Farkas D, Gauldie J, et al. Transient overexpression of Gremlin results in epithelial activation and reversible fibrosis in rat lungs. Am J Respir Cell Mol Biol 2011; 44: 870-878.

27 Gupte VV, Ramasamy SK, Reddy R, et al. Overexpression of fibroblast growth factor-10 during both inflammatory and fibrotic phases attenuates bleomycin-induced pulmonary fibrosis in mice. Am J Respir Crit Care Med 2009; 180: $424-436$

28 Soroosh A, Albeiroti S, West GA, et al. Crohn's disease fibroblasts overproduce the novel protein KIAA1199 to create proinflammatory hyaluronan fragments. Cell Mol Gastroenterol Hepatol 2016; 2: 358-368 e354.

29 Li L, Yan LH, Manoj S, et al. Central role of CEMIP in tumorigenesis and its potential as therapeutic target. J Cancer 2017; 8: 2238-2246.

30 McKee CM, Penno MB, Cowman M, et al. Hyaluronan (HA) fragments induce chemokine gene expression in alveolar macrophages. The role of HA size and CD44. J Clin Invest 1996; 98: 2403-2413.

31 Teder P, Vandivier RW, Jiang D, et al. Resolution of lung inflammation by CD44. Science 2002; 296: 155-158.

32 Balaji S, Wang X, King A, et al. Interleukin-10-mediated regenerative postnatal tissue repair is dependent on regulation of hyaluronan metabolism via fibroblast-specific STAT3 signaling. FASEB J 2017; 31: 868-881.

33 Liang J, Zhang Y, Xie T, et al. Hyaluronan and TLR4 promote surfactant-protein-C-positive alveolar progenitor cell renewal and prevent severe pulmonary fibrosis in mice. Nat Med 2016; 22: 1285-1293.

34 Savani RC, Hou G, Liu P, et al. A role for hyaluronan in macrophage accumulation and collagen deposition after bleomycin-induced lung injury. Am J Respir Cell Mol Biol 2000; 23: 475-484.

35 Swigris JJ, Brown KK. The role of endothelin-1 in the pathogenesis of idiopathic pulmonary fibrosis. BioDrugs 2010; 24: 49-54.

36 Konigshoff M, Dumitrascu R, Udalov S, et al. Increased expression of 5-hydroxytryptamine2A/B receptors in idiopathic pulmonary fibrosis: a rationale for therapeutic intervention. Thorax 2010; 65: 949-955. 